Vietnam Journal of Mechanics, VAST, Vol. 34, No. 2 (2012), pp. $113-121$

\title{
HOMOGENIZATION OF AN INTERFACE HIGHLY OSCILLATING BETWEEN TWO CONCENTRIC ELLIPSES
}

\author{
Do Xuan Tung, Pham Chi Vinh, Nguyen Kim Tung \\ Hanoi University of Science, $V N U$
}

\begin{abstract}
The main purpose of this paper is to find the homogenized equation and the associate continuity conditions in the explicit form of a boundary-value problem in a twodimensional domain with an interface oscillating rapidly between two concentric ellipses. This boundary-value problem originates from various problems in practical applications. By the homogenization method and following the technique presented recently by Vinh and Tung [P. C. Vinh and D. X. Tung, Mech. Res. Comm. 37 (2010), 285-288; P. C. Vinh, D. X. Tung, ASME J. Appl. Mech., 78 (2011), 041014-1; P. C. Vinh and D. X. Tung, Acta Mech. 218 (2011), 333-348], the homogenized equation and the associate continuity conditions in the explicit form are derived.
\end{abstract}

Key words: Interfaces oscillating highly between two concentric ellipses, homogenization method, homogenized equation.

\section{INTRODUCTION}

Boundary-value problems in domains with rough boundaries or interfaces is closely related to various practical problems such as scattering of elastic waves at rough boundaries and interfaces [1], transmission and reflection of waves on rough interfaces $[2,3,4]$, mechanical problems concerning the plates with densely spaced stiffeners [5], flows over rough walls [6] and so on. When the amplitude (height) of the roughness is much small comparison with its period, the problems are usually analyzed by perturbation methods. When the amplitude is much large than its period, i.e. the boundaries and interfaces are very rough, the homogenization method $[7,8,9]$ is required.

In [10], Nevard and Keller investigated a boundary-value problem in a two-dimensional domain with an interface highly oscillating between two straight lines (see Fig. 1), namely

$$
\begin{gathered}
\left(\sigma U_{x}\right)_{x}+\left(\sigma U_{z}\right)_{z}-\lambda U=f(x, z),(x, z) \notin L \\
{[U]_{L}=0 ; \quad\left[\sigma U_{n}\right]_{L}=0}
\end{gathered}
$$




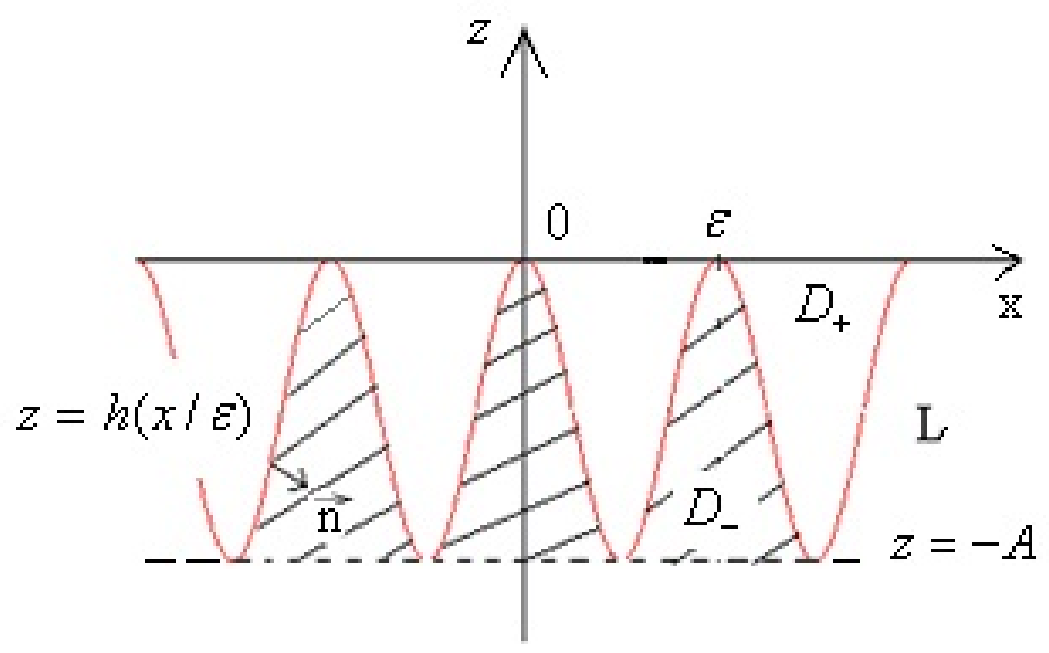

Fig. 1. Two-dimensional domains $D_{+}$and $D_{-}$have a very rough interface L expressed by equation $z=h(x / \varepsilon)=h(y)$, where $h(y)$ is a periodic function with period 1 . The curve $\mathrm{L}$ highly oscillates between the straight lines $z=0$ and $z=-A(A>0)$.

where $\phi_{x}:=\partial \phi / \partial x, \phi_{z}:=\partial \phi / \partial z, \ldots, \phi_{n}=\phi_{x} n_{x}+\phi_{z} n_{z}, n_{x}, n_{z}$ are the components of the unit normal to the curve $L, U=U(x, z)$ is unknown, $f(x, z), \sigma, \lambda$ are given and

$$
\sigma, \lambda=\left\{\begin{array}{l}
\sigma_{+}, \lambda_{+} \text {for }(x, z) \in D_{+} \\
\sigma_{-}, \lambda_{-} \text {for }(x, z) \in D_{-}
\end{array}\right.
$$

$\sigma_{+}, \sigma_{-}, \lambda_{+}, \lambda_{-}$are constant and $[w]_{L}=w_{+}-w_{-}$on $L$. The interface $L$ is called a very rough interface.

By using the homogenization method the authors derived the homogenized equation and the associate continuity conditions in the explicit form. This problem was then considered in a two-dimensional domain with an interface highly oscillating between two concentric circles [10], and the corresponding homogenized equation and associate continuity conditions were also found.

The main purpose of the present paper is to extend the results of Nevard and Keller to the case when the interface oscillates rapidly between two concentric ellipses. To do that we first write Eq. (1) and the continuity conditions (2) in the generalized polar coordinates. Then, by employing the homogenization method and following the technique presented recently in Refs. $[11,12,13]$, the authors obtain the homogenized equation and the associate continuity conditions in the explicit form. 


\section{INTERFACES HIGHLY OSCILLATING BETWEEN TO CONCENTRIC ELLIPSES}

Now we consider the boundary-value problem (1)-(2) in $D=D_{+} \cup L \cup D_{-}$, where $D_{+}, D_{-}$are separated by the interface $L$ expressed by

$$
\left\{\begin{array}{l}
x=a_{1} h(\theta / \varepsilon) \cos \theta \\
z=b_{1} h(\theta / \varepsilon) \sin \theta
\end{array} \quad, 0 \leq \theta<2 \pi\right.
$$

where $a_{1}, b_{1}$ are given positive numbers, $0<\varepsilon=2 \pi / N<<1, N$ is a sufficiently large positive integer number, $h(\varphi), \varphi=\theta / \varepsilon$, is a periodic function with period 1 and its minimum value is 1 and its maximum value is $k=a_{2}: a_{1}>1, a_{2}$ is a given positive number. One can see that the (closed) curve $L$ oscillates highly between two concentric

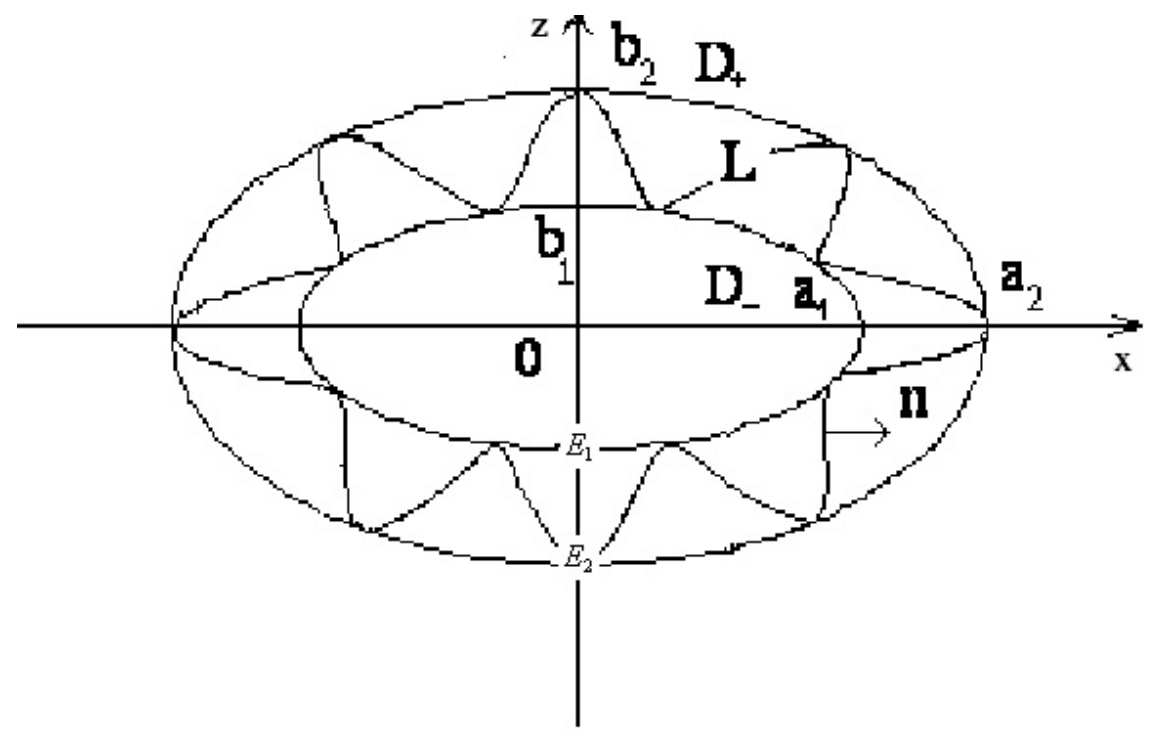

Fig. 2. The interface $L$, expressed by (4), oscillates highly between two concentric ellipses $E_{1}$ and $E_{2}$ defined by (5).

ellipses $E_{1}$ and $E_{2}$ (see Fig. 2) defined respectively by

$$
\frac{x^{2}}{a_{1}^{2}}+\frac{z^{2}}{b_{1}^{2}}=1 \text { and } \frac{x^{2}}{a_{2}^{2}}+\frac{z^{2}}{b_{2}^{2}}=1, a_{2}: a_{1}=b_{2}: b_{1}=k>1
$$

The domain $D_{-}\left(D_{+}\right)$lies inside (outside) the closed curve $L$. We also assume that any ellipse $x^{2} / a^{2}+z^{2} / b^{2}=1, a_{1}<a<a_{2}, b_{1}<b<b_{2}, a: b=a_{1}: b_{1}=a_{2}: b_{2}$, has exactly two intersections with the curve $L$. From (4) we have

$$
n_{x}: n_{z}=-z^{\prime}(\theta): x^{\prime}(\theta)
$$

the continuity condition (2) thus takes the form

$$
[U]_{L}=0,\left[\sigma\left\{b_{1}\left(\varepsilon^{-1} h^{\prime} \sin \theta+h \cos \theta\right) U_{x}-a_{1}\left(\varepsilon^{-1} h^{\prime} \cos \theta-h \sin \theta\right) U_{z}\right\}\right]_{L}=0
$$




\section{Remark 1:}

Through the mapping

$$
X=x / a_{1}, Z=z / b_{1}
$$

the curve $L$ belong to the plane $(x, z)$ is mapped to the curve $L^{*}$ belong to the plane $(X, Z)$ defined by

$$
X=h(\theta / \varepsilon) \cos \theta, Z=h(\theta / \varepsilon) \cos \theta, 0 \leq \theta<2 \pi
$$

that oscillates highly between two concentric circles $X^{2}+Z^{2}=1$ and $X^{2}+Z^{2}=k^{2}$, denoted by $E_{1}^{*}$ and $E_{2}^{*}$ (see Fig. 3). These circles are respectively the images of the ellipses

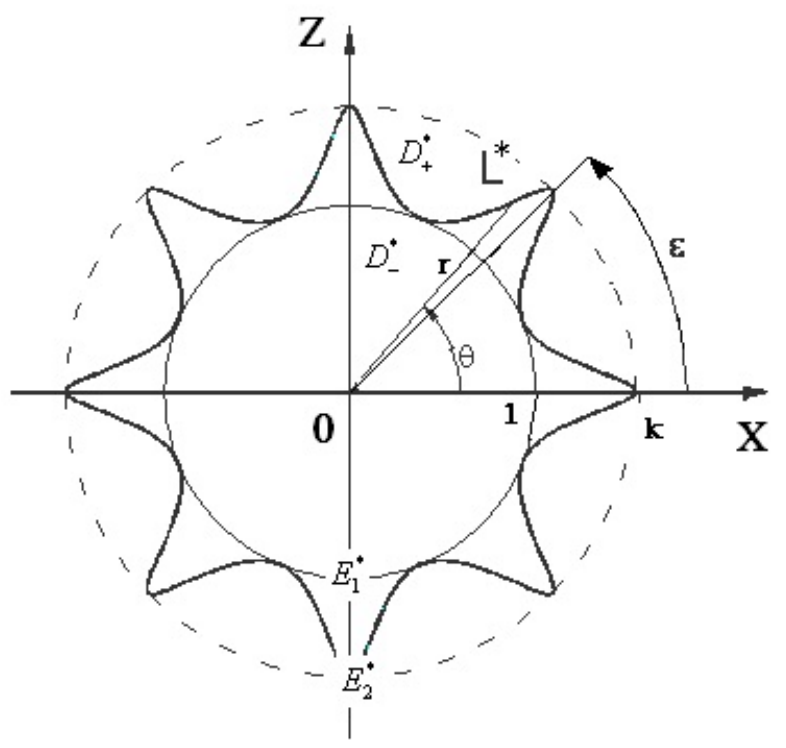

Fig. 3. The curve $L^{*}$, expressed by $r=h(\theta / \varepsilon)$, oscillates rapidly between two concentric circles $E_{1}: X^{2}+Z^{2}=1$ and $E_{2}: X^{2}+Z^{2}=k^{2}$.

$E_{1}$ and $E_{2}$ through the mapping (8). In terms of the polar coordinates $r, \theta$ of the plane $(X, Z)$ (i. e. $X=r \cos \theta, Z=r \sin \theta)$, the curve $L^{*}$ is expressed by $r=h(\theta / \varepsilon)$. Since $x=a_{1} r \cos \theta, z=b_{1} r \sin \theta, r, \theta$ are the generalized polar coordinates of the plane $(x, z)$.

Our purpose is to study asymptotic behavior of the boundary-value problem (1), (7) when $\varepsilon \rightarrow 0$. In particular, we want to find the explicit homogenized equation of the problem $(1),(7)$ and the associate boundary conditions in terms of the variables $r, \theta$. Note that $\sigma$ and $\lambda$ are defined by (3).

From Remark 1, it is convenient to study the problem (1), (7) in the plane $(X, Z)$. By $D_{+}^{*}$ and $D_{-}^{*}$ we denote the images of $D_{+}$and $D_{-}$through the mapping (8) (see Fig.3). The domains $D_{+}^{*}$ and $D_{-}^{*}$ are separated by $L^{*}$ which highly oscillates between two concentric 
circles: $E_{1}^{*}$ with radius 1 and $E_{2}^{*}$ with radius $k$, and it is expressed by equation $r=h(\theta / \varepsilon)$. In terms of the variables $X, Z$, Eq. (1) becomes

$$
\frac{1}{a_{1}^{2}}\left(\sigma U_{X}\right)_{X}+\frac{1}{b_{1}^{2}}\left(\sigma U_{Z}\right)_{Z}-\lambda U=f(X, Z),(X, Z) \notin L^{*}
$$

Since $X=r \cos \theta, Z=r \sin \theta$, we have

$$
\left\{\begin{array}{l}
\frac{\partial}{\partial X}=\cos \theta \frac{\partial}{\partial r}-\frac{\sin \theta}{r} \frac{\partial}{\partial \theta} \\
\frac{\partial}{\partial Z}=\sin \theta \frac{\partial}{\partial r}+\frac{\cos \theta}{r} \frac{\partial}{\partial \theta}
\end{array}\right.
$$

Introducing (11) into (10) yields

$$
k_{1}\left(\sigma U_{r}\right)_{r}+\frac{k_{2}}{r^{2}}\left(\sigma U_{\theta}\right)_{\theta}-\frac{k_{3}}{r}\left[\left(\sigma U_{\theta}\right)_{r}+\left(\sigma U_{r}\right)_{\theta}\right]+\frac{k_{2}}{r} \sigma U_{r}+\frac{2 k_{3}}{r^{2}} \sigma U_{\theta}-\lambda U=f, r \neq h(\theta / \varepsilon)
$$

where

$$
k_{1}=\frac{\cos ^{2} \theta}{a_{1}^{2}}+\frac{\sin ^{2} \theta}{b_{1}^{2}}, k_{2}=\frac{\sin ^{2} \theta}{a_{1}^{2}}+\frac{\cos ^{2} \theta}{b_{1}^{2}}, k_{3}=\left(\frac{1}{a_{1}^{2}}-\frac{1}{b_{1}^{2}}\right) \sin \theta \cos \theta
$$

Similarly, in terms of the polar coordinates $(r, \theta)$ of the plane $(X, Z)$, the continuity condition (7) is of the form

$$
[U]_{L^{*}}=0,\left[\sigma U_{r}\left(k_{1}+\frac{h^{\prime}}{\varepsilon r} k_{3}\right)-\sigma U_{\theta}\left(\frac{k_{3}}{r}+\frac{h^{\prime}}{\varepsilon r^{2}} k_{2}\right)\right]_{L^{*}}=0
$$

\section{EXPLICIT HOMOGENIZED EQUATION}

Following Bensoussan et al. [7], Sanchez-Palencia [8] we can suppose that $U(r, \theta, \varepsilon)=$ $u(r, \theta, \varphi, \varepsilon)$. Then we have

$$
U_{\theta}=u_{\theta}+\frac{1}{\varepsilon} u_{\varphi}
$$

Substituting (15) into Eq. (12) and the continuity condition $(14)_{2}$ leads to

$$
\begin{array}{r}
\frac{k_{2}}{\varepsilon^{2} r^{2}}\left(\sigma u_{\varphi}\right)_{\varphi}+\frac{k_{2}}{\varepsilon r^{2}}\left[\left(\sigma u_{\varphi}\right)_{\theta}+\left(\sigma u_{\theta}\right)_{\varphi}\right]-\frac{k_{3}}{\varepsilon r}\left[\left(\sigma u_{\varphi}\right)_{r}+\left(\sigma u_{r}\right)_{\varphi}\right]+\frac{2 k_{3}}{\varepsilon r^{2}} \sigma u_{\varphi}+k_{1}\left(\sigma u_{r}\right)_{r} \\
+\frac{k_{2}}{r^{2}}\left(\sigma u_{\theta}\right)_{\theta}-\frac{k_{3}}{r}\left[\left(\sigma u_{r}\right)_{\theta}+\left(\sigma u_{\theta}\right)_{r}\right]+\frac{k_{2}}{r} \sigma u_{r}+\frac{2 k_{3}}{r^{2}} \sigma u_{\theta}-\lambda u=f, r \neq h(\varphi) \\
{[u]_{L^{*}}=0, \quad\left[\sigma u_{r}\left(k_{1}+\frac{h^{\prime}}{\varepsilon r} k_{3}\right)-\sigma u_{\theta}\left(\frac{k_{3}}{r}+\frac{h^{\prime}}{\varepsilon r^{2}} k_{2}\right)-\sigma u_{\varphi}\left(\frac{k_{3}}{\varepsilon r}+\frac{h^{\prime}}{\varepsilon^{2} r^{2}} k_{2}\right)\right]_{L^{*}}=0}
\end{array}
$$

Following Vinh and Tung [11, 12, 13], $u(r, \theta, \varphi, \varepsilon)$ is expressed as follows

$$
\begin{aligned}
u=V & +\varepsilon\left(N^{1} V+N^{11} V_{r}+N^{12} V_{\theta}\right) \\
& +\varepsilon^{2}\left(N^{2} V+N^{21} V_{r}+N^{22} V_{\theta}+N^{211} V_{r r}+N^{212} V_{r \theta}+N^{222} V_{\theta \theta}\right)+O\left(\varepsilon^{3}\right)
\end{aligned}
$$

where $V=V(\theta, r)$ (being independent of $\varphi$ ), $N^{i}, N^{i j}, N^{i j k}$ are functions of $\varphi$ and $r$ (not depending on $\theta$ ) and they are $\varphi$-periodic with period 1 . The functions $N^{i}, N^{i j}, N^{i j k}$ are chosen so that the equation (16) and the continuity conditions (17) are satisfied. 
From (18), it is clear that the continuity $(17)_{1}$ is satisfied if

$$
\left[N^{i}\right]_{L^{*}}=0,\left[N^{i j}\right]_{L^{*}}=0,\left[N^{i j k}\right]_{L^{*}}=0
$$

Substituting (18) into (16) and $(17)_{2}$ yields equations which we call $\left(e_{1}\right)$ and $\left(e_{2}\right)$, respectively. In order to make the coefficients of $\varepsilon^{-1}$ and $\varepsilon^{0}$ of $\left(e_{1}\right)$ zero we take

$$
\frac{k_{2}}{r^{2}}\left(\sigma N_{\varphi}^{1}\right)_{\varphi} V+\left(\frac{k_{2}}{r^{2}} \sigma N_{\varphi}^{11}-\frac{k_{3}}{r} \sigma\right)_{\varphi} V_{r}+\frac{k_{2}}{r^{2}}\left[\sigma\left(N_{\varphi}^{12}+1\right)\right]_{\varphi} V_{\theta}=0
$$

and

$$
\begin{aligned}
& \left\{\frac{k_{2}}{r^{2}}\left(\sigma N_{\varphi}^{2}\right)_{\varphi}-\frac{k_{3}}{r}\left[\left(\sigma N_{\varphi}^{1}\right)_{r}+\left(\sigma N_{r}^{1}\right)_{\varphi}\right]+\frac{2 k_{3}}{r^{2}} \sigma N_{\varphi}^{1}\right\} V+ \\
& \left\{\frac{k_{2}}{r^{2}}\left(\sigma N_{\varphi}^{21}\right)_{\varphi}-\frac{k_{3}}{r}\left[\sigma N_{\varphi}^{1}+\left(\sigma N^{1}\right)_{\varphi}+\left(\sigma N_{\varphi}^{11}\right)_{r}+\left(\sigma N_{r}^{11}\right)_{\varphi}\right]+\frac{2 k_{3}}{r^{2}} \sigma N_{\varphi}^{11}+\frac{k_{2}}{r} \sigma\right\} V_{r}+ \\
& \left\{\frac{k_{2}}{r^{2}}\left[\left(\sigma N^{1}\right)_{\varphi}+\sigma N_{\varphi}^{1}\right]-\frac{k_{3}}{r}\left[\left(\sigma N_{\varphi}^{12}\right)_{r}+\left(\sigma N_{r}^{12}\right)_{\varphi}\right]+\frac{2 k_{3}}{r^{2}} \sigma\left(N_{\varphi}^{12}+1\right)+\frac{k_{2}}{r^{2}}\left(\sigma N_{\varphi}^{22}\right)_{\varphi}\right\} V_{\theta} \\
& +\left\{\frac{k_{2}}{r^{2}}\left[\left(\sigma N^{11}\right)_{\varphi}+\sigma N_{\varphi}^{11}+\left(\sigma N_{\varphi}^{212}\right)_{\varphi}\right]-\frac{k_{3}}{r}\left[\sigma\left(N_{\varphi}^{12}+1\right)+\left(\sigma N^{12}\right)_{\varphi}\right]\right\} V_{r \theta}+ \\
& \left\{\frac{k_{2}}{r^{2}}\left(\sigma N_{\varphi}^{211}\right)_{\varphi}-\frac{k_{3}}{r}\left[\sigma N_{\varphi}^{11}+\left(\sigma N^{11}\right)_{\varphi}\right]\right\} V_{r r}+ \\
& \frac{k_{2}}{r^{2}}\left\{\left(\sigma N_{\varphi}^{222}\right)_{\varphi}+\left(\sigma N^{12}\right)_{\varphi}+\sigma\left(N_{\varphi}^{12}+1\right)\right\} V_{\theta \theta}+ \\
& k_{1}\left(\sigma V_{r}\right)_{r}-\frac{k_{3}}{r}\left(\sigma V_{\theta}\right)_{r}-\lambda V=f
\end{aligned}
$$

Vanishing the coefficients of $\varepsilon^{-1}$ and $\varepsilon^{0}$ of $\left(e_{2}\right)$ gives

$$
\frac{h^{\prime} k_{2}}{r^{2}}\left[\sigma N_{\varphi}^{1}\right]_{L^{*}} V+\frac{h^{\prime}}{r}\left[\frac{k_{2}}{r} \sigma N_{\varphi}^{11}-k_{3} \sigma\right]_{L^{*}} V_{r}+\frac{h^{\prime} k_{2}}{r^{2}}\left[\sigma\left(N_{\varphi}^{12}+1\right)\right]_{L^{*}} V_{\theta}=0
$$

and

$$
\begin{aligned}
& {\left[\sigma\left\{\frac{h^{\prime} k_{3}}{r} N_{r}^{1}-\frac{k_{3}}{r} N_{\varphi}^{1}-\frac{h^{\prime} k_{2}}{r^{2}} N_{\varphi}^{2}\right\}\right]_{L^{*}} V+} \\
& {\left[\sigma\left\{k_{1}+\frac{h^{\prime} k_{3}}{r}\left(N^{1}+N_{r}^{11}\right)-\frac{k_{3}}{r} N_{\varphi}^{11}-\frac{h^{\prime} k_{2}}{r^{2}} N_{\varphi}^{21}\right\}\right]_{L^{*}} V_{r}+} \\
& {\left[\sigma\left\{\frac{h^{\prime} k_{3}}{r} N_{r}^{12}-\frac{k_{3}}{r}\left(N_{\varphi}^{12}+1\right)-\frac{h^{\prime} k_{2}}{r^{2}}\left(N_{\varphi}^{22}+N^{1}\right)\right\}\right]_{L^{*}} V_{\theta}+} \\
& {\left[\frac{k_{3} h^{\prime}}{r} \sigma N^{12}-\frac{h^{\prime} k_{2}}{r^{2}} \sigma\left(N^{11}+N_{\varphi}^{212}\right)\right]_{L^{*}} V_{r \theta}+\left[\sigma\left(\frac{h^{\prime} k_{3}}{r} N^{11}-\frac{h^{\prime} k_{2}}{r^{2}} N_{\varphi}^{211}\right)\right]_{L^{*}} V_{r r}-} \\
& \frac{h^{\prime} k_{2}}{r^{2}}\left[\sigma\left(N^{12}+N_{\varphi}^{222}\right)\right]_{L^{*}} V_{\theta \theta}=0
\end{aligned}
$$

To make (20) and (22) satisfied, the functions $N^{1}, N^{11}, N^{12}$ are chosen as follows (taking into account (19))

$$
\begin{gathered}
\left(\sigma N_{\varphi}^{1}\right)_{\varphi}=0,0<\varphi<1, \varphi \neq \varphi_{1}, \varphi_{2} \\
{\left[\sigma N_{\varphi}^{1}\right]_{L^{*}}=0,\left[N^{1}\right]_{L^{*}}=0, N^{1}(0)=N^{1}(1)=0}
\end{gathered}
$$




$$
\begin{gathered}
\left(\sigma\left[N_{\varphi}^{12}+1\right]\right)_{\varphi}=0,0<\varphi<1, \varphi \neq \varphi_{1}, \varphi_{2} \\
{\left[\sigma\left(N_{\varphi}^{12}+1\right)\right]_{L^{*}}=0,\left[N^{12}\right]_{L^{*}}=0, N^{12}(0)=N^{12}(1)=0} \\
\left(\frac{k_{2}}{r} \sigma N_{\varphi}^{11}-k_{3} \sigma\right)_{\varphi}=0,0<\varphi<1, \varphi \neq \varphi_{1}, \varphi_{2} \\
{\left[\left(\frac{k_{2}}{r} \sigma N_{\varphi}^{11}-k_{3} \sigma\right)\right]_{L^{*}}=0,\left[N^{11}\right]_{L^{*}}=0, N^{11}(0)=N^{11}(1)=0}
\end{gathered}
$$

where $\varphi_{1}$ and $\varphi_{2}\left(0<\varphi_{1}<\varphi_{2}<1\right)$ are two roots of the equation $h(\varphi)=r$ for $\varphi$ in the interval $(0,1)$ in which $r$, as a parameter, belong to the domain $(1, k)$. The functions $\varphi_{1}(r)$ and $\varphi_{2}(r)$ are two inverse branches of the function $r=h(\varphi)$. Note that, from a mentioned above assumption, any circle $X^{2}+Z^{2}=c^{2}(1<c<k)$ has exactly two intersections with the curve $L^{*}$.

From (24)-(26), it is not difficult to show that

$$
\begin{gathered}
N^{1} \equiv 0,\left\langle\sigma N_{\varphi}^{11}\right\rangle=\frac{k_{3} r}{k_{2}}\left(\langle\sigma\rangle-\left\langle\sigma^{-1}\right\rangle^{-1}\right),\left\langle\sigma\left(N_{\varphi}^{12}+1\right)\right\rangle=\left\langle\sigma^{-1}\right\rangle^{-1} \\
\sigma N_{\varphi}^{11}=\frac{k_{3} r}{k_{2}}\left(\sigma-\left\langle\sigma^{-1}\right\rangle^{-1}\right), \sigma N_{\varphi}^{12}=\left\langle\sigma^{-1}\right\rangle^{-1}-\sigma
\end{gathered}
$$

where

$$
\langle g\rangle=\int_{0}^{1} g d \varphi=\left(\varphi_{2}-\varphi_{1}\right) g_{+}+\left(1+\varphi_{1}-\varphi_{2}\right) g_{-}
$$

$g_{+}$and $g_{-}$are the values of $g$ in $D_{+}^{*}$ and $D_{-}^{*}$, respectively.

In order to make (23) satisfy we take (noting that $N^{1} \equiv 0$ by $(27)_{1}$ )

$$
\begin{aligned}
& {\left[\sigma N_{\varphi}^{2}\right]_{L^{*}}=0,\left[\sigma\left\{k_{1}+\frac{h^{\prime} k_{3}}{r} N_{r}^{11}-\frac{k_{3}}{r} N_{\varphi}^{11}-\frac{h^{\prime} k_{2}}{r^{2}} N_{\varphi}^{21}\right\}\right]_{L^{*}}=0} \\
& {\left[\sigma\left\{\frac{h^{\prime} k_{3}}{r} N_{r}^{12}-\frac{k_{3}}{r}\left(N_{\varphi}^{12}+1\right)-\frac{h^{\prime} k_{2}}{r^{2}} N_{\varphi}^{22}\right\}\right]_{L^{*}}=0} \\
& {\left[\frac{k_{3}}{r} \sigma N^{12}-\frac{k_{2}}{r^{2}} \sigma\left(N^{11}+N_{\varphi}^{212}\right)\right]_{L^{*}}=0,\left[\sigma\left(\frac{k_{3}}{r} N^{11}-\frac{k_{2}}{r^{2}} N_{\varphi}^{211}\right)\right]_{L^{*}}=0} \\
& {\left[\sigma\left(N^{12}+N_{\varphi}^{222}\right)\right]_{L^{*}}=0}
\end{aligned}
$$

By integrating equation (21) along the circle $r=$ const, $1<r<k$ from $\varphi=0$ to $\varphi=1$, and taking into account (27) and (29), we obtain

$$
\begin{aligned}
& {\left[\frac{1}{r}\left(\frac{k_{3}^{2}}{k_{2}}+k_{2}\right)\langle\sigma\rangle-\frac{1}{r} \frac{k_{3}^{2}}{k_{2}}\left\langle\sigma^{-1}\right\rangle^{-1}+\left(k_{1}-\frac{k_{3}^{2}}{k_{2}}\right)\langle\sigma\rangle_{r}+\frac{k_{3}^{2}}{k_{2}}\left(\left\langle\sigma^{-1}\right\rangle^{-1}\right)_{r}\right] V_{r}+} \\
& {\left[\frac{2 k_{3}}{r^{2}}\left\langle\sigma^{-1}\right\rangle^{-1}-\frac{k_{3}}{r}\left(\left\langle\sigma^{-1}\right\rangle^{-1}\right)_{r}\right]} \\
& \quad V_{\theta}-\frac{2 k_{3}}{r}\left\langle\sigma^{-1}\right\rangle^{-1} V_{r \theta}+\left[\left(k_{1}-\frac{k_{3}^{2}}{k_{2}}\right)\langle\sigma\rangle+\frac{k_{3}^{2}}{k_{2}}\left\langle\sigma^{-1}\right\rangle^{-1}\right] V_{r r} \\
& +\frac{k_{2}}{r^{2}}\left\langle\sigma^{-1}\right\rangle^{-1} V_{\theta \theta}-\langle\lambda\rangle V=f, 1<r<k, 0 \leq \theta<2 \pi
\end{aligned}
$$

This is the desired explicit homogenized equation that defies in the domain bounded by two ellipses $E_{1}, E_{2}$ in the plane $(x, z)$. In the domains $0<r<1,0 \leq \theta<2 \pi$ and 
$r>k, 0 \leq \theta<2 \pi$ must be satisfied the following equations

$$
\begin{gathered}
\sigma_{-}\left(k_{1} V_{r r}-\frac{2 k_{3}}{r} V_{r \theta}+\frac{k_{2}}{r^{2}} V_{\theta \theta}+\frac{k_{2}}{r} V_{r}+\frac{2 k_{3}}{r^{2}} V_{\theta}\right)-\lambda_{-} V=f, 0<r<1,0 \leq \theta<2 \pi \\
\sigma_{+}\left(k_{1} V_{r r}-\frac{2 k_{3}}{r} V_{r \theta}+\frac{k_{2}}{r^{2}} V_{\theta \theta}+\frac{k_{2}}{r} V_{r}+\frac{2 k_{3}}{r^{2}} V_{\theta}\right)-\lambda_{+} V=f, r>k, 0 \leq \theta<2 \pi
\end{gathered}
$$

which originate from Eq. (12). In addition to Eqs. (30), (31) and (32) the continuity conditions on the ellipses $E_{1}$ and $E_{2}$ are required, namely

$$
[V]_{E_{i}}=0,\left[\left(\left\{k_{1}-\frac{k_{3}^{2}}{k_{2}}\right\}\langle\sigma\rangle+\frac{k_{3}^{2}}{k_{2}}\left\langle\sigma^{-1}\right\rangle^{-1}\right) V_{r}-\frac{k_{3}}{r}\left\langle\sigma^{-1}\right\rangle^{-1} V_{\theta}\right]_{E_{i}}=0, i=1,2 .
$$

The ellipses $E_{1}$ and $E_{2}$ correspond to $r=1$ and $r=k$, respectively. Note that the continuity condition $(33)_{2}$ originates from the condition: $\left[\sigma U_{n}\right]_{E_{i}}=0, i=1,2, n$ is the normal unit to $E_{i}$, or equivalently

$$
\left[\sigma\left(k_{1} u_{r}-\frac{k_{3}}{r}\left\{u_{\theta}+\varepsilon^{-1} u_{\varphi}\right\}\right)\right]_{E_{i}}=0, i=1,2 .
$$

Introducing (18) into (34) yields an equation denoted by $\left(e_{3}\right)$. Equating to zero the coefficient of $\varepsilon^{0}$ of $\left(e_{3}\right)$ provides

$$
\left[\left(k_{1} \sigma-\frac{k_{3}}{r} \sigma N_{\varphi}^{11}\right) V_{r}-\frac{k_{3}}{r} \sigma\left(N_{\varphi}^{12}+1\right) V_{\theta}\right]_{E_{i}}=0, i=1,2 .
$$

Integrating (35) along the lines $r=1$ and $r=k$ from $\varphi=0$ to $\varphi=1$ and using (27) we arrive at $(33)_{2}$.

When the ellipses $E_{1}$ and $E_{2}$ become the circles with radii $a_{1}\left(=b_{1}\right)$ and $a_{2}\left(=b_{2}\right)$, i. e. $k_{1}=k_{2}=1, k_{3}=0$, Eq. (30) and the continuity (33) simplify respectively to

$$
\frac{1}{r}\left(r\langle\sigma\rangle V_{r}\right)_{r}+\frac{1}{r^{2}}\left\langle\sigma^{-1}\right\rangle^{-1} V_{\theta \theta}-\langle\lambda\rangle V=f
$$

and

$$
[V]_{E_{i}}=0,\left[\langle\sigma\rangle V_{r}\right]_{E_{i}}=0, i=1,2 .
$$

They coincide with Eq. (4.6) and the associate continuity condition in Ref.[10] obtained by Nevard and Keller by a different way.

When the materials of $D_{+}$and $D_{-}$are the same, i. e.: $\sigma_{+}=\sigma_{-}=\sigma, \lambda_{+}=\lambda_{-}=\lambda$, Eq. (30) becomes

$$
\sigma\left(k_{1} V_{r r}-\frac{2 k_{3}}{r} V_{r \theta}+\frac{k_{2}}{r^{2}} V_{\theta \theta}+\frac{k_{2}}{r} V_{r}+\frac{2 k_{3}}{r^{2}} V_{\theta}\right)-\lambda V=f
$$

that coincides with Eq. (31) and Eq. (32). 


\section{CONCLUSIONS}

By using techniques of the homogenization method the authors derive the explicit homogenized equation and the associate continuity conditions of a boundary-value problem, originating from various problems in practical applications, in a two-dimensional domain with an interface highly oscillating between two concentric ellipses. The obtained results recover the ones derived by Nevard and Keller [10] for the interface oscillating highly between two concentric circles. Since the obtained homogenized equation and the associate continuity conditions are explicit, they are convenient in use.

\section{ACKNOWLEDGEMENT}

The work was supported by the Vietnam National Foundation For Science and Technology Development (NAFOSTED).

\section{REFERENCES}

[1] Zaki, K. A., Neureuther, A. R., Scattering from a perfectly conducting surface with a sinusoidal hight profile: TE polarization, IEEE Trans. Atenn. Propag., 19(2), (1971), 208 - 214.

[2] Talbot, J. R. S., Tichener, J. B., Willis, J. R., The reflection of electromagnetic waves from very rough interface, Wave Motion, 12, (1990), 245 - 260.

[3] Singh, S. S., Tomar, S. K., Quassi-P-waves at a corrugated interface between two dissimilar monoclinic elastic half-spaces, Int. J. Solids Struct., 44, (2007), 197 - 228.

[4] Singh, S. S., Tomar, S. K., qP-wave at a corrugated interface between two dissimilar prestressed elastic half-spaces, J. Sound Vibr., 317, (2008), 687 - 708.

[5] Cheng, K. T., Olhoff, N., An investigation concerning optimal design of solid elastic plates, Int. J. Solids Struct., 17, (1981), 795 - 810.

[6] Achdow, Y., Pironncau, O., Valentin, F., Effective boundary conditions for laminar flows over rough boundaries, J. Comput. Phys., 147, (1998), 187 - 218.

[7] Bensoussan, A., Lions, J. B., Papanicolaou, J., Asymptotic analysics for periodic structures, North- Holland, Amsterdam, (1978).

[8] Sanchez-Palencia E., Nonhomogeneous media and vibration theory, Lecture Notes in Phys., Springer-Verlag, Heidelberg, 127, (1980).

[9] Bakhvalov, N., Panasenco, G., Homogenization: averaging of processes in periodic media: mathematical problems of the mechanics of composite materials, Kluwer Acar. Publ., Dordrecht Boston London, (1989).

[10] Nevard J. and Keller J. B., Homogenization of rough boundaries and interface, SAM J. Appl. Math., 57(6), (1997), 1660 - 1686.

[11] Vinh, P. C. and Tung, D. X., Homogenized equations of the linear elasticity in two-dimensional domains with very rough interfaces, Mech. Res. Comm., 37, (2010), 285 - 288.

[12] Vinh, P. C., Tung, D. X., Homogenization of rough two-dimensional interfaces separating two anisotropic solids, ASME J. Appl. Mech., 78(4), (2011), 41014-41021.

[13] Vinh, P. C. and Tung, D.X., Homogenized equations of the linear elasticity theory in twodimensional domains with interfaces highly oscillating between two circles, Acta Mech., 218, (2011), $333-348$. 